\title{
Effects of Noise-Induced Hearing Loss at Young Age on Voice Onset Time and Gap-in-Noise Representations in Adult Cat Primary Auditory Cortex
}

\author{
NaOtaka Aizawa ${ }^{1,2}$ and Jos J. Eggermont ${ }^{1,2}$ \\ ${ }^{1}$ Department of Physiology and Biophysics, University of Calgary, Calgary, Alberta, Canada \\ ${ }^{2}$ Department of Psychology, University of Calgary, 2500 University Drive N.W., Calgary, Alberta, Canada, T2N 1N4
}

Received: 5 July 2005; Accepted: 9 December 2005; Online publication: 12 January 2006

\begin{abstract}
Here we show that mild hearing loss induced by noise exposure in early age causes a decrease in neural temporal resolution when measured in adulthood. We investigated the effect of this chronic hearing loss on the representation of a voice onset time (VOT) and a gap-duration continuum in primary auditory cortex (AI) in cats, which were exposed at the age of 6 weeks to a 120-dB SPL, $5-\mathrm{kHz} 1 / 3$ octave noise band for $2 \mathrm{~h}$. The resulting hearing loss measured using auditory brainstem responses and cortical multiunit thresholds at 4-6 months of age was 20-40 dB between 1 and $32 \mathrm{kHz}$. Multiple single-unit activity was recorded in seven noise-exposed cats and nine control cats related to the presentation of a/ba/-/ $\mathrm{pa} /$ continuum in which VOT was varied in 5-ms step from 0 to $70 \mathrm{~ms}$. We also obtained data for noise bursts with gaps, of duration equal to the VOT, embedded in noise $5 \mathrm{~ms}$ after the onset. Both stimuli were presented at $65 \mathrm{~dB}$ SPL. Minimum VOT and early-gap duration were defined as the lowest value in which an on-response, significantly above the spontaneous activity, to both the leading and trailing noise bursts or vowel was obtained. The mild chronic noise-induced hearing loss increased the minimum detectable VOT and gap duration by $10 \mathrm{~ms}$. We also analyzed the maximum firing rate $\left(\mathrm{FR}_{\max }\right)$ and the latency of the responses as a function of VOT and
\end{abstract}

Correspondence to: Jos J. Eggermont - Department of Psychology • University of Calgary $\bullet 2500$ University Drive N.W., Calgary, Alberta, Canada T2N 1N4. Telephone: (403) 220-5214; fax: (403) 282-8249; email: eggermon@ucalgary.ca gap duration and found a significant reduction in the $\mathrm{FR}_{\max }$ to the trailing noise burst for gap durations above $50 \mathrm{~ms}$. This suggests that mild hearing loss acquired in early age may affect cortical temporal processing in adulthood.

Keywords: cat, primary auditory cortex, multiunit activity, noise trauma, hearing loss, voice onset time, gap thresholds

\section{INTRODUCTION}

Voice onset time (VOT), the time interval between the consonant stop closure and the onset of voicing, is an important determinant of the categorical perception boundary, that value of the VOT at which $50 \%$ of the phonemes are correctly labeled. Thus, phoneme confusion, e.g., between voiced (e.g., /ba/) and voiceless (e.g., /pa/) consonant-vowel phonemes, might potentially arise from changes in the neural representation of VOT, as this may affect the perceptual boundaries, albeit indirectly. High-frequency hearing loss of sufficient severity (Revoile et al. 1987) interferes with speech intelligibility resulting specifically in consonant confusion. It is therefore possible that such hearing loss will affect the neural representation of VOT. However, young children with mild to moderate hearing loss $(\leq 55 \mathrm{~dB})$ showed no difference in their phonetic boundary between the phonemes / ba/ and / pa/ presented at $25 \mathrm{~dB}$ above threshold (Johnson et al. 1984), suggesting that either the neural representation of this boundary in auditory cortex is unaffected or the children learn to adjust their perceptual boundary. 
Neural VOT thresholds in auditory cortex are defined here as the minimum VOT that evokes a double on-response in evoked local field potentials or multiunit activity (Steinschneider et al. 1994, 1995, $1999,2003)$ or single neuron or neuron-cluster responses (Eggermont 1995, 1999, 2000). Neural VOT thresholds may be related to perceptual boundaries, but, as scalp-recorded evoked potential data have shown (Sharma et al. 2000), they are not identical to these boundaries. Previously, we have shown that in the first few hours after noise exposure in adult cats, the VOT threshold for a $/ \mathrm{ba} /-/ \mathrm{pa} /$ continuum shifted to higher values and that the peak firing rate-VOT function became shallower, suggesting that the discrimination boundary between / ba/ and / pa/ became less well defined (Tomita et al. 2004). This study also showed a comparable change in the threshold for a double-onset response to a noise burst with a gap inserted $5 \mathrm{~ms}$ after the onset: a socalled "early gap."

It is likely that immediately after a noise trauma, the neurons in the auditory cortex are subject to a different combination of excitation and inhibition strengths than several months later when a new balance has emerged (Noreña et al. 2003; Noreña and Eggermont 2005). We also have to consider that behaviorally measured hearing loss induced by noise exposure in young rats was larger than that in the adults, and there was also relatively more hearing loss for the lower frequencies in young animals compared to adults (Rybalko and Syka 2001).

Here we investigate the effect of noise trauma induced in 6-week-old kittens on temporal processing and on the cortical representation of VOT when these cats reached adulthood. For that purpose, we compared results using a / ba/-/pa/-like continuum, but with the spectra of the initial noise burst and the vowel transformed upward to be able to activate the high-CF neurons, with an "early gap" in noise stimulus (Eggermont 1999). We found that the mild noise-induced hearing loss of about 20-30 dB increased the minimum VOT and minimum early-gap duration by about $10 \mathrm{~ms}$, twice as large as previously observed within a few hours after noise trauma in adult cats (Tomita et al. 2004).

\section{METHODS}

\section{Animal preparation}

All animals were deeply anesthetized with the administration of $25 \mathrm{mg} / \mathrm{kg}$ of ketamine hydrochloride and $20 \mathrm{mg} / \mathrm{kg}$ of sodium pentobarbital, injected intramuscularly. A mixture of $0.2 \mathrm{ml}$ of acepromazine $(0.25 \mathrm{mg} / \mathrm{ml})$ and $0.8 \mathrm{ml}$ of atropine methyl nitrate (25 mg/ml) was administered subcutaneously at approximately $0.25 \mathrm{ml} / \mathrm{kg}$ body weight. Lidocaine $(20 \mathrm{mg} / \mathrm{ml})$ was injected subcutaneously prior to incision. The tissue overlying the right temporal lobe was removed, and the dura was resected to expose the area bounded by anterior and posterior ectosylvian sulci. The cat was then secured with one screw cemented on the head without any other restraint. The wound margins were infused every $2 \mathrm{~h}$ with lidocaine, and additional acepromazine/atropine mixture was administered every $2 \mathrm{~h}$. The ketamine dose to maintain a state of areflexive anesthesia was on average $7.4 \mathrm{mg} / \mathrm{kg} \mathrm{h}$ (range $3-12 \mathrm{mg} / \mathrm{kg} \mathrm{h}$ ).

The care and the use of animals reported in this study were approved (\#BI 2001-021) and reviewed on a yearly basis by the Life and Environmental Sciences Animal Care Committee of the University of Calgary. All animals were maintained and handled according to the guidelines set by the Canadian Council of Animal Care.

The ear contralateral to the speaker (ipsilateral to the cortex recorded from) was filled with an ear mold substance (Dur-A-Sil, Insta-Mold Products). In one normal hearing cat (not included in the present series), we found that ear molds inserted attenuated, on average, $32 \mathrm{~dB}$ in the frequency range above $600 \mathrm{~Hz}$.

\section{Peripheral threshold estimation}

Peripheral hearing sensitivity was determined from auditory brainstem response (ABR) thresholds in an anechoic room. The use of $\mathrm{ABR}$ in cats has been validated previously by comparison with thresholds of auditory nerve fibers (Ngan and May 2001). Tone pips with frequencies of $3,4,6,8,12,16,24$, and 32 $\mathrm{kHz}$ were presented (monaurally, because the contralateral ear is filled with ear mold) at a rate of $10 / \mathrm{s}$ in an anechoic room from a speaker placed $45^{\circ}$ from the midline and at $55-\mathrm{cm}$ distance from the cat's head. Calibration and monitoring of the sound field were performed using a Brüel and Kjäer (type 4134) microphone placed above the animal's head and facing the loud speaker. ABRs were recorded, with needle electrodes in ipsilateral and contralateral muscles covering both mastoids, in response to gamma-function-shaped tone pips with an effective (50\% from peak) duration of $15 \mathrm{~ms}$. The signals were amplified between 300 and $3000 \mathrm{~Hz}$ using a DAM 500 (WPI) differential amplifier and averaged with a Brüel and Kjäer (type 2034) dual spectral analyzer in the signal enhancement mode. Artifact rejection and local lidocaine infusion were used to avoid contamination of the ABR by muscle action potentials. We used the component in the ABR that was detectable at the lowest sound levels, and this was nearly always wave 4. At high-intensity levels, 20-50 
averages sufficed, but at near threshold values, 200300 averages were obtained and repeated once. The level was decreased (step size was $10 \mathrm{~dB}$ ) for as long as responses were reproducible. Threshold was defined as the highest sound level that yielded a nonreproducible response $+5 \mathrm{~dB}$ SPL. The peripheral hearing loss was estimated by comparing ABR thresholds obtained in the exposed cats with respect to mean ABR thresholds in the control group.

\section{Acoustic stimulus presentation}

Stimuli were generated in MATLAB $^{\circledR}$ and were transferred to the DSP boards of a TDT-2 (Tucker Davis Technologies) sound delivery system. Acoustic stimuli were presented in an anechoic room from a speaker system [Fostex RM765 in combination with a Realistic Super-Tweeter that produced a flat spectrum $( \pm 5 \mathrm{~dB})$ up to $40 \mathrm{kHz}$ measured at the cat's head] placed approximately $30^{\circ}$ from the midline into the contralateral field, about $50 \mathrm{~cm}$ from the cat's left ear. Calibration and monitoring of the sound field were accomplished with a condenser microphone (Brüel and Kjäer 4134) placed above the animal's head, facing the speaker and a measuring amplifier (Brüel and Kjäer 2636). The characteristic frequency and tuning properties of individual neurons were determined using the same gamma-tone pips (Eggermont 1996a) as used for ABR recordings. These tone pips have a half-peak amplitude duration of $15 \mathrm{~ms}$ and a gamma-function-shaped envelope. Twenty-seven tone pips with frequencies covering five octaves were presented at eight different intensities (i.e., mainly between 5 and $75 \mathrm{~dB}$ SPL in 10-dB steps), and each intensity-frequency combination was repeated five times. The presentation rate was $4 / \mathrm{s}$.

After the frequency tuning properties of the cells at each electrode were determined, the $/ \mathrm{ba} /-/ \mathrm{pa} /$ continuum with VOTs ranging from 0 to $70 \mathrm{~ms}$ in steps of $5 \mathrm{~ms}$ was presented in random order as described previously (Eggermont 1995). These phonemes were generated with a parallel/cascade Klattsynthesizer KLSYN88a using a $20-\mathrm{kHz}$ sampling frequency. The total duration of the stimulus was $250 \mathrm{~ms}$, regardless of the duration of the VOT, and the onset noise burst was $5 \mathrm{~ms}$ in duration (leading noise burst). The peak level of the onset noise burst was about $10 \mathrm{~dB}$ lower than that of the vowel. The dominant frequency ranges for the vowel part were $\mathrm{F} 0=120 \mathrm{~Hz}, \mathrm{~F} 1=700 \mathrm{~Hz}$, and F2 $=1200 \mathrm{~Hz}$. The fundamental frequency started at $120 \mathrm{~Hz}$, remained at that value for $100 \mathrm{~ms}$, and then dropped from there to $100 \mathrm{~Hz}$ at the end of the vowel. F1 started at $512 \mathrm{~Hz}$ and increased in $25 \mathrm{~ms}$ to $700 \mathrm{~Hz}, \mathrm{~F} 2$ started at $1019 \mathrm{~Hz}$ and increased in $25 \mathrm{~ms}$ to $1200 \mathrm{~Hz}$, and F3 changed in the same time span from 2153 to 2600
Hz. To allow activating high-CF neurons, the vowel spectrum was transformed (after interpolation and resampling at $40 \mathrm{kHz}$ ) so that the upper edge of the $\mathrm{F} 2$ band was at $8 \mathrm{kHz}$. The vowel duration, amplitude modulation, and glottal pulse periodicity were not changed. The phonemes were presented once every 2 s. The only parameter that changed in the continuum was the VOT; formant transition durations were not altered. Twenty repetitions were presented at each VOT value so that the entire stimulus sequence lasted $600 \mathrm{~s}$.

Gaps ranging from 5 to $70 \mathrm{~ms}$ in duration were placed in two positions in wideband noise bursts of $1 \mathrm{~s}$ in duration that were presented once per $3 \mathrm{~s}$ in random order. The first position, the "early gap," started $5 \mathrm{~ms}$ after the noise burst onset, and the second position, the "late gap," was placed $500 \mathrm{~ms}$ after the noise burst onset. Here, in contrast to the stimuli in the $/ \mathrm{ba} /-/ \mathrm{pa} /$ continuum, the level of the leading and trailing noise bursts was the same. Only results for the early gap will be presented as they relate to the VOT results (Eggermont 1999). Each early-gap stimulus consisted of $1 \mathrm{~s}$ of noise followed by $2 \mathrm{~s}$ of silence and was presented 15 times. All stimuli were presented at peak $(/ \mathrm{ba} /-/ \mathrm{pa} /)$ or RMS (gap-in-noise) levels of $65 \mathrm{~dB}$ SPL.

\section{Acoustic trauma}

To introduce a high-frequency hearing loss, kittens at 6 weeks after birth were exposed in the anechoic room for $2 \mathrm{~h}$ to a $1 / 3$ octave band of noise centered at $5 \mathrm{kHz}$. Measured at the cat's head, the noise had a level of around $120 \mathrm{~dB}$ SPL, a level that has previously been shown to introduce about 20- to 40$\mathrm{dB}$ high-frequency hearing loss in adult animals (Noreña and Eggermont 2005). The sound was presented by a high-power amplifier (Samson Servo 240) and loudspeaker (Yorkville $120 \mathrm{~W}$ ) placed 75 $\mathrm{cm}$ away from the animals. During this exposure, the awake animals were confined in a small cage and facing the loudspeaker. Nearly all cats fell asleep during the exposure, and none showed signs of discomfort. After the exposure, the cats were placed in a quiet room until they were at least 22 weeks old.

\section{Recording and spike separation procedure}

Two arrays of eight microelectrodes (Frederic Haer Corp.), each with impedances between 1 and $2 \mathrm{M} \Omega$, were used. The electrodes were arranged in a $4 \times 2$ configuration with interelectrode distance within rows and columns equal to $0.5 \mathrm{~mm}$. Each electrode array was oriented such that most electrodes were touching the cortical surface and then were manually and independently advanced using a Narishige M101 
hydraulic microdrive (one drive for each array). The signals were processed by a TDT-Pentusa multichannel data acquisition system (filter bandwidth of 300 $\mathrm{Hz}-10 \mathrm{kHz}$ ). Spike sorting was performed off-line using a semiautomated procedure based on principal component analysis and $K$-means clustering implemented in MATLAB. The spike times and waveforms were stored. The multiunit data presented in this paper were composed only of units with a regular spike wave form that are likely generated dominantly by pyramidal cells (Eggermont 1996a).

\section{Data analysis}

To assess frequency-tuning properties, the peak number of action potentials in the poststimulus time histogram (PSTH, 5-ms bins) calculated over the first $100 \mathrm{~ms}$ after gamma-tone presentation was estimated. The results were calculated per stimulus intensity and were combined into an intensity-frequency-rate profile from which tuning curves, rate-intensity functions, and isointensity rate-frequency contours could be derived (Eggermont 1996a) using routines implemented in MATLAB. The frequency-tuning curve was defined for a firing rate at $25 \%$ of the maximum peak firing rate. This was about $10-20 \%$ above the background firing rate, but as the latter was dependent on the level of stimulus-induced suppression, the tuning curve criterion based on a percentage of peak firing rate was preferred over that based on increase over background activity.

PSTHs with a 5-ms bin width were also used to represent the recordings for the $/ \mathrm{ba} /-/ \mathrm{pa} /$ continuum and gap stimuli. From these PSTHs, both the peak latency and the spike count $\left(\mathrm{FR}_{\max }\right)$, optionally normalized on the peak response to the initial noise burst, were calculated. The minimum neural VOT (MNVOT) was defined as the minimum VOT at which neural responses are time-locked to both the burst onset and the onset of voicing. Similarly, the minimum neural gap threshold (MNGT) for the "early" gap stimulus was defined as the minimum gap duration at which neural responses are time-locked to both the leading and the trailing noise burst onsets. Data for units with both an on-response to the leading noise burst and the vowel or trailing noise burst were averaged across the population of neurons. Initially, this was performed separately for frequency regions below and above $6 \mathrm{kHz}$, but because there was no significant difference between them, the averages were obtained over the entire collection of recordings.

The MNVOT and MNGT were obtained from a comparison of the population response to the vowel or trailing noise burst and the population spontaneous activity just before the leading noise burst. We defined the MNVOT and MNGT as the response to the trailing burst or vowel, for the smallest VOT or gap duration, that was significantly higher than the value in the bin representing spontaneous activity in the time window of 10-35 ms (five bins) after trailing burst or vowel onset. Because five time bins covered the response duration to the trailing noise burst (Figs. 3 and 4), and the response in each of those bins was compared to the one bin representing spontaneous activity, a Bonferroni correction was applied, and the significance level was set at $p<0.01$. All statistical analyses were performed using Statview $5^{\circledR}$.

\section{RESULTS}

Recordings were made from the right primary auditory cortex in 16 cats [9 control cats (201 recordings) and 7 cats (125 recordings) exposed to noise at 6 weeks of age]. The age of cats at the time of recording ranged from 152 to 218 days (mean = 171.3 days, $\mathrm{SD}=23.0$ days) in the control group and from 140 to 182 days $($ mean $=157.3$ days, $\mathrm{SD}=17.3$ days) in the noise-trauma group. In the noise-trauma group, the time between the noise trauma and recording ranged from 93 to 135 days (mean = 108.5 days, $\mathrm{SD}=18.2$ days). The weight of cats was between 2130 and $3500 \mathrm{~g}$ (mean $=3098 \mathrm{~g}, \mathrm{SD}=$ $518.9 \mathrm{~g}$ ) in the control group and from 1930 to $2850 \mathrm{~g}$ (mean $=2475 \mathrm{~g}, \mathrm{SD}=302.7 \mathrm{~g}$ ) in the noisetrauma group. There were no significant differences in the age and weights between the two groups.

\section{Peripheral threshold}

The amount of peripheral hearing loss induced by the band-pass noise exposure was established by ABR. Thresholds from ABR were obtained from eight control cats and five noise-trauma cats completely; in the other cats, only partial results were obtained because of low signal-to-noise ratio. Figure 1 shows the differences of the mean threshold between the control and the noise-trauma group at each frequency for the animals that provided complete ABR recordings. The hearing loss measured was significantly different from that in the control group over the entire frequency range.

\section{Frequency-tuning properties}

Figure $2 \mathrm{a}$ and $\mathrm{b}$ shows the tuning curves of an MU recording for a control cat and a noise-trauma cat. Contour lines are shown for 75, 50, and 25\%, whereby the lower contour is considered here as the neuron's frequency-tuning curve. These figures are typical for the two groups in the CF region between 6 


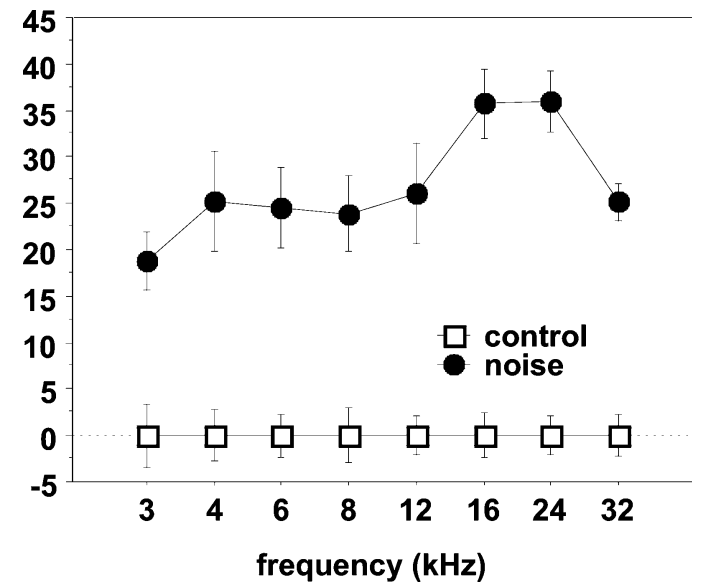

FIG. 1. ABR threshold shifts for the noise-exposed group compared to the control group. The ABR thresholds for the control group were set at zero. The bars indicate standard error. The threshold shifts were significant over the frequency range measured.

and $24 \mathrm{kHz}$; the threshold at $\mathrm{CF}$ is higher, and the tuning curve at $20 \mathrm{~dB}$ above the threshold is broader in the noise-trauma group compared with the control group. Figure 2c shows the MU thresholds as a function of CF. The mean threshold in the noise-
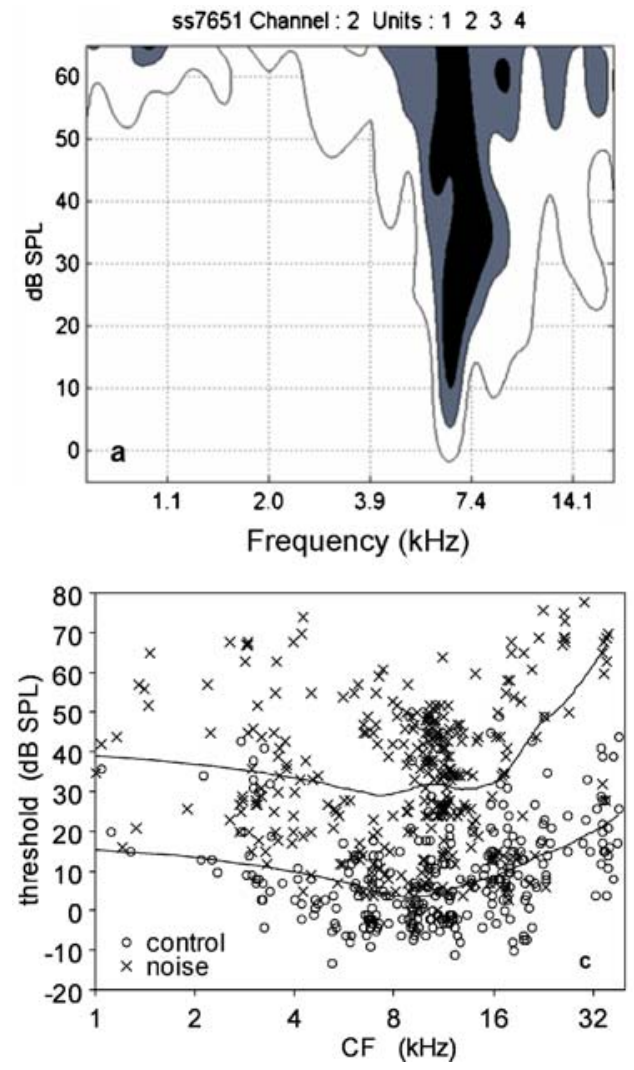

FIG. 2. Parts a and b show representative frequency tuning curves from the 10- to 20-kHz CF range for a control animal (a) and a noiseexposed animal (b). Part (c) shows the thresholds at CF for the control and the noise-exposed group; locally weighted regression lines are drawn in. The exposed group shows a nearly CF- trauma group is, on average, $24 \mathrm{~dB}$ SPL higher than that in the control group, which is comparable to the results obtained with ABR. There is hardly any $\mathrm{CF}$ dependence for this threshold difference below 16 $\mathrm{kHz}$; above this $\mathrm{CF}$, the hearing loss increased up to $45 \mathrm{~dB}$. Figure $2 \mathrm{~d}$ shows the frequency-tuning curve bandwidth at $20 \mathrm{~dB}$ above threshold at $\mathrm{CF}\left(\mathrm{BW}_{20} \mathrm{~dB}\right)$ as a function of $\mathrm{CF}$. Mean values of $\mathrm{BW}_{20} \mathrm{~dB}$ for the noise-trauma group and the control group were 1.50 and 1.28 octave, which is a significant difference $(p<$ $0.01)$. However, when the $\mathrm{CF}$ was divided into three subgroups $(\mathrm{CF}<10,10 \leq \mathrm{CF}<20$, and $\mathrm{CF} \geq 20 \mathrm{kHz})$, only the subgroup with $\mathrm{CFs}$ between 10 and $20 \mathrm{kHz}$ showed a significant difference between the noise trauma and the control group, and the other subgroups showed no significant differences $(\mathrm{CF}<$ $10 \mathrm{kHz}, p=0.69 ; 10 \leq \mathrm{CF}<20 \mathrm{kHz}, p<0.01 ; \mathrm{CF} \geq 20$ $\mathrm{kHz}, p=0.16)$.

\section{Individual example}

Figure 3 shows neural responses for a control-cat recording obtained from the same electrode site for the presentation of the $/ \mathrm{ba} /-/ \mathrm{pa} /$ continuum $(\mathrm{a}-\mathrm{c})$
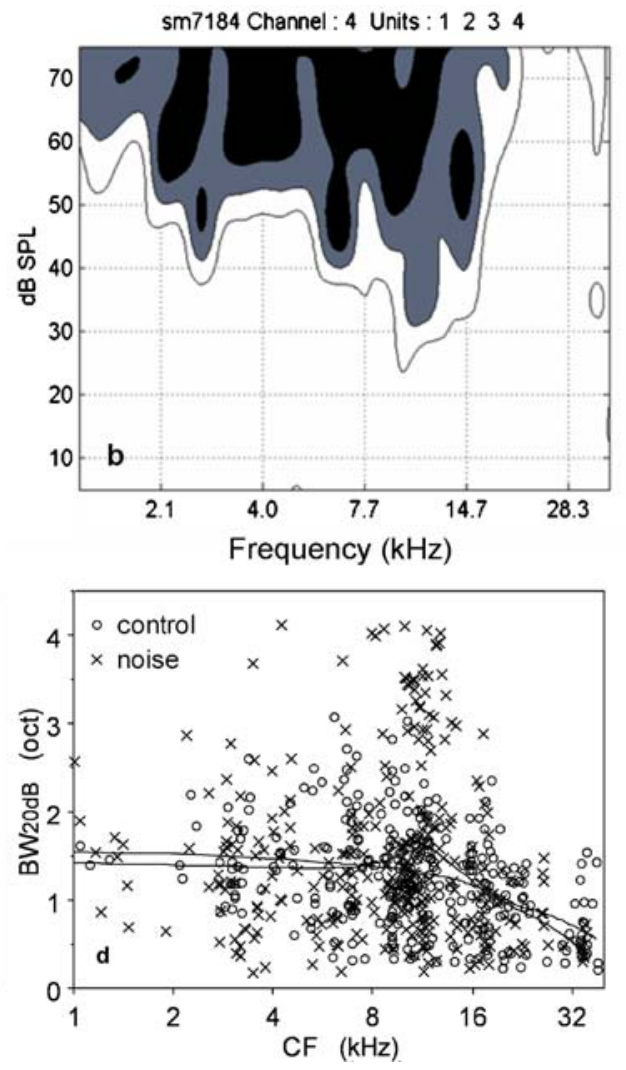

independent threshold increase of $24 \mathrm{~dB}$ between 1 and $16 \mathrm{kHz}$. For higher CFs, the threshold shift increases to $45 \mathrm{~dB}$ at $32 \mathrm{kHz}$. Part (d) shows the frequency-tuning curve bandwidth at $20 \mathrm{~dB}$ above threshold; only in the CF range between 10 and $20 \mathrm{kHz}$ is the bandwidth significantly different between the two groups. 

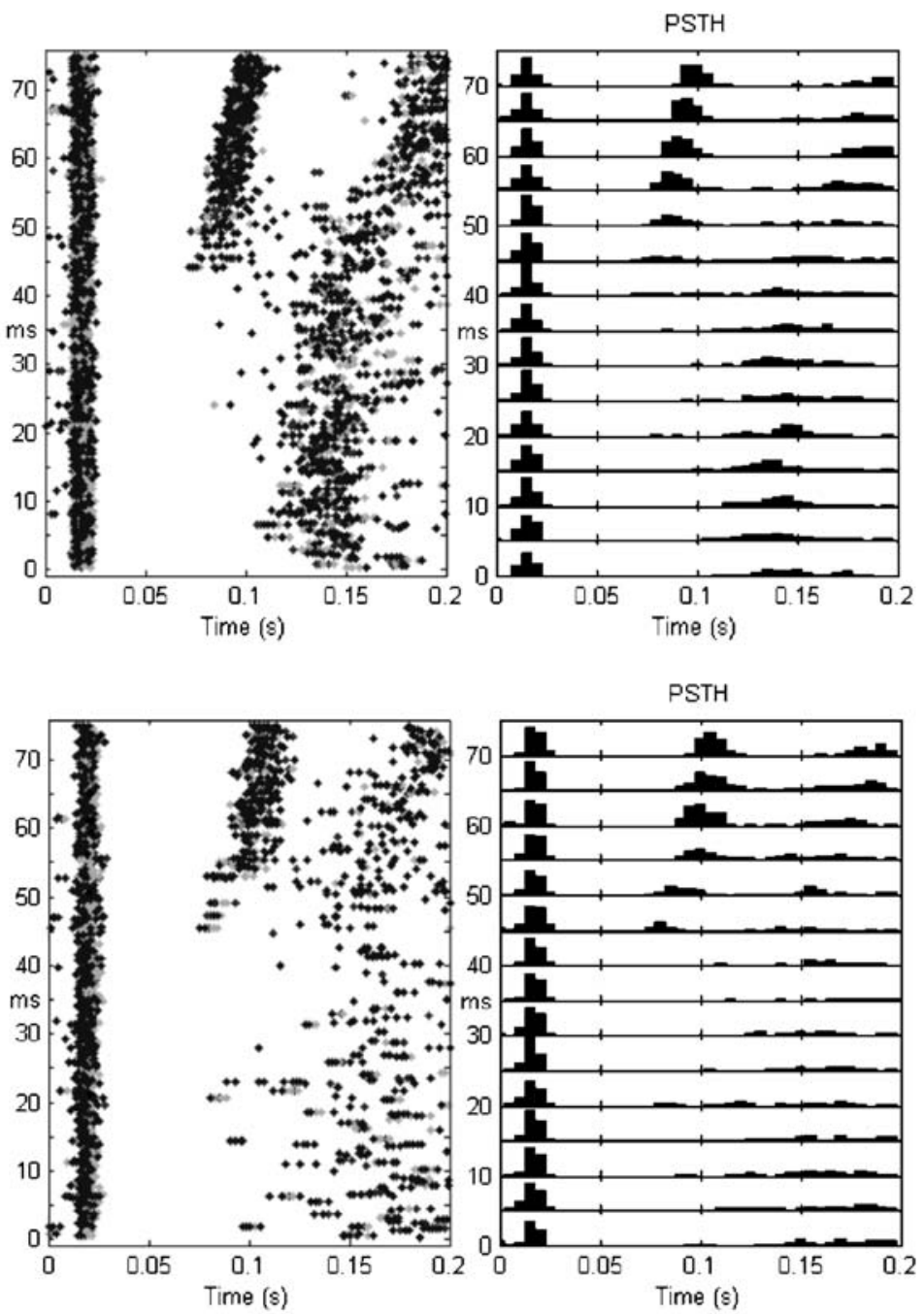
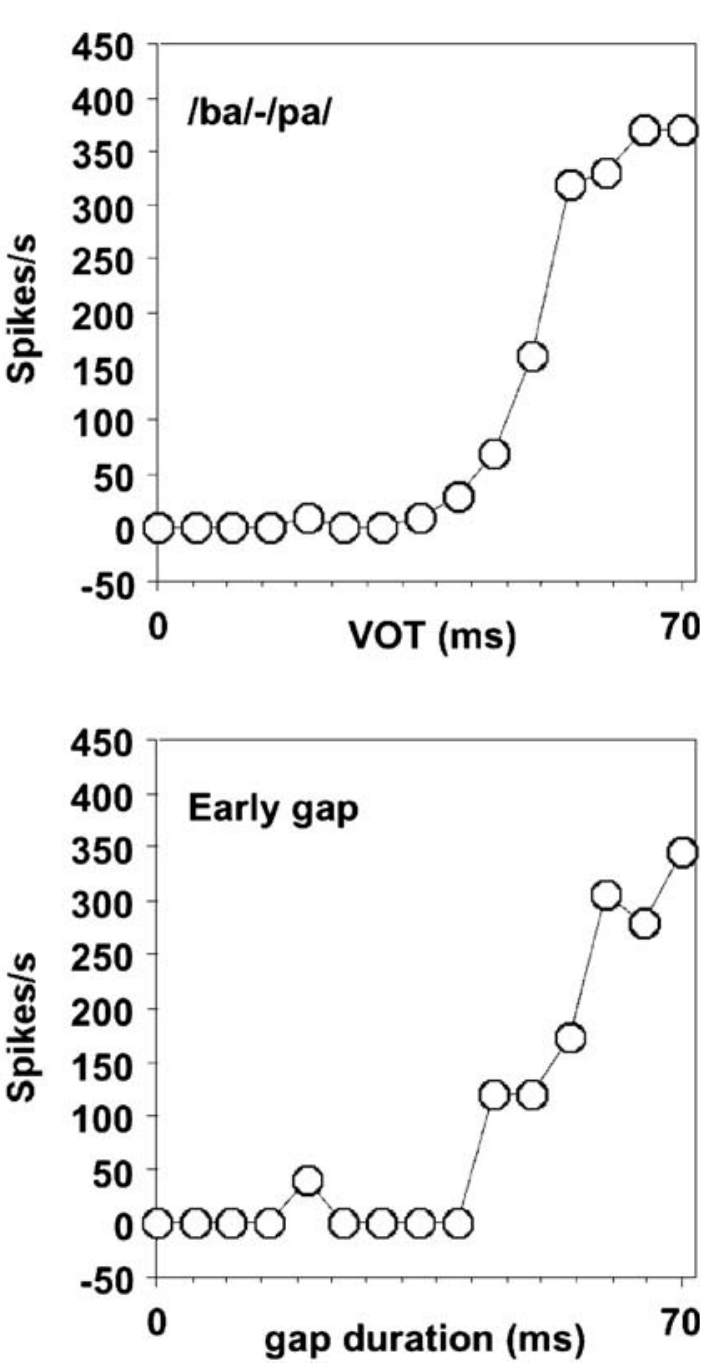

FIG. 3. Dot displays (left column), poststimulus-time histograms (middle column), and peak firing rate $\left(\mathrm{FR}_{\max }\right)$ as a function of VOT or early-gap duration (right column). The upper row shows data for a

and the "early-gap" stimuli (d-f), both at $65 \mathrm{~dB}$ SPL. Here, and in the following, we will not distinguish between the original $/ \mathrm{ba} /-/ \mathrm{pa} /$ stimulus and the frequency-transformed one. The original $/ \mathrm{ba} /-/ \mathrm{pa} /$ was used for CFs below $2.5 \mathrm{kHz}$, and the transformed one was used for all recordings with higher CFs. Panels (a) and (d) show dot displays that are organized vertically according to VOT or gap duration and horizontally for time since leading noise burst onset. Panels (b) and (e) represent the PSTHs (5-ms bin size) of the data shown in panels (a) and (d), respectively. Panels (c) and (f) show the maximum firing rate $\left(\mathrm{FR}_{\max }\right)$ per bin in the PSTH for the response to the vowel or trailing noise burst as a function of VOT or gap duration. One notes first that the responses related to the presentation of the $/ \mathrm{ba} /-/ \mathrm{pa} /$ continuum are similar to those related to the presentation of the "early-gap" stimuli. The /ba/-/pa/ continuum; the lower row shows data for a gap-in-noise continuum recorded at the same site. Different colors in the dot display indicate different spike-sorted units.

MNVOT and MNGT were estimated from the dot displays at $45 \mathrm{~ms}$. There are very few spikes evoked by stimuli with VOT or the gap duration below $40 \mathrm{~ms}$. For higher VOT or gap durations, there is an approximately sigmoidally increasing $\mathrm{FR}_{\max }$. In Figure 3 left column, rebound responses following the response to the initial noise burst at latencies around $130 \mathrm{~ms}$ were seen for short VOTs. For longer VOTs, the rebound response followed the response to the vowel with a slightly shorter latency.

\section{Group data}

Because the $\mathrm{FR}_{\max }$ of the responses to the leading noise burst or to the vowel or the trailing noise burst was quite variable, we normalized this peak firing rate. The normalized firing rate for each recording was obtained by dividing the $\mathrm{FR}_{\max }$ in each PSTH bin 

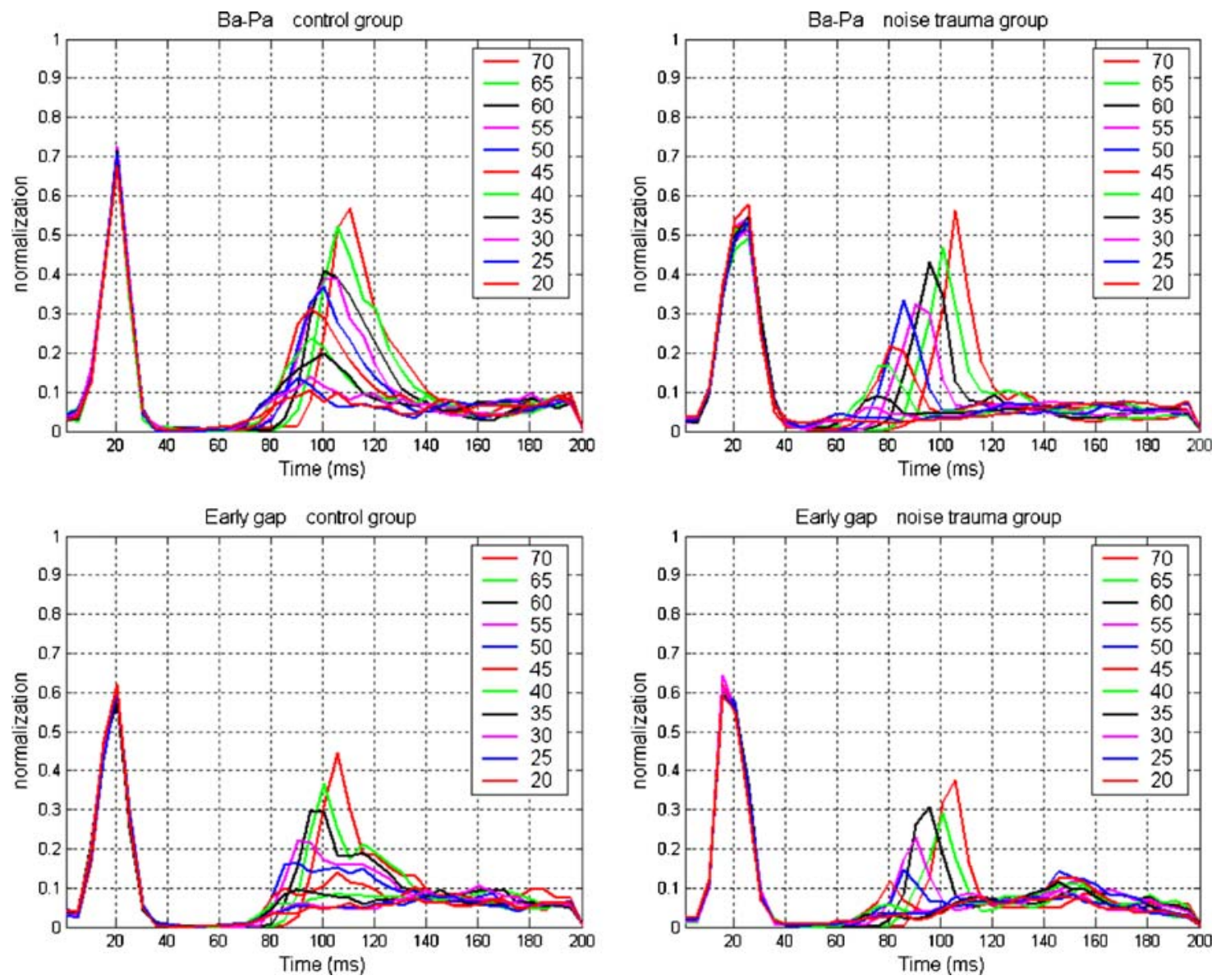

FIG. 4. Population PSTHs for controls (left column, $N=100$ ) and noise-exposed animals (right column, $N=62$ ) for the $/ \mathrm{ba} /-/ \mathrm{pa} /$ continuum (top row) and the gap-in-noise continuum (bottom row). Before averaging, the responses were normalized on the largest on-

by the peak PSTH rate to the leading noise burst across all VOTs or gap durations in that recording. Figure 4 shows the population average of these normalized firing rates as a function of VOT or gap duration. In the control group, the spontaneous firings are suppressed over 20-30 ms following the response to the leading noise burst. In the noiseexposed group, the suppression lasts either shorter (about $20 \mathrm{~ms}$ for the early-gap group) or is less complete (for the $/ \mathrm{ba} /-/ \mathrm{pa} / \mathrm{stimuli}$ ). The number of recordings in each group, together with the mean

response to the leading noise burst. Responses to the leading noise burst overlap for all VOT or gap-duration conditions; those for the vowel or trailing noise burst depend on VOT or gap duration (indicated by color).

and $\mathrm{SD}$ of $\mathrm{FR}_{\max }$ to the leading noise burst, is shown in Table $1 . \mathrm{FR}_{\max }$ to the leading noise burst for the / $\mathrm{ba} /-/ \mathrm{pa} /$ continuum was significantly reduced by the noise trauma $(p<0.0001)$.

The latency of the peak response to the leading burst was 15-25 ms, and the latency of the peak response to the vowel or the trailing noise burst followed the increasing VOT or gap duration. The activity in the first bin $(0-5 \mathrm{~ms})$ was taken to represent the spontaneous activity and was used for calculating the significance of responses to the vowel

TABLE 1

Number of recordings, peak firing rates $\left(\mathrm{FR}_{\max }\right)$ to the leading noise burst, and estimated minimum VOT (MNVOT) and minimum gap duration (MNGT) in control and noise-exposed animals

\begin{tabular}{|c|c|c|c|c|c|c|}
\hline & \multicolumn{3}{|c|}{ /ba/-/pa/ } & \multicolumn{3}{|c|}{ Early gap } \\
\hline & $N$ & $F R_{\max }(s p / s)$ & MNVOT (ms) & $N$ & $F R_{\max }(\mathrm{sp} / \mathrm{s})$ & MNGT (ms) \\
\hline Control & 100 & $214 \pm 116$ & 20 & 101 & $201 \pm 120$ & 30 \\
\hline Noise trauma & 63 & $150 \pm 103$ & 30 & 62 & $225 \pm 142$ & 40 \\
\hline
\end{tabular}



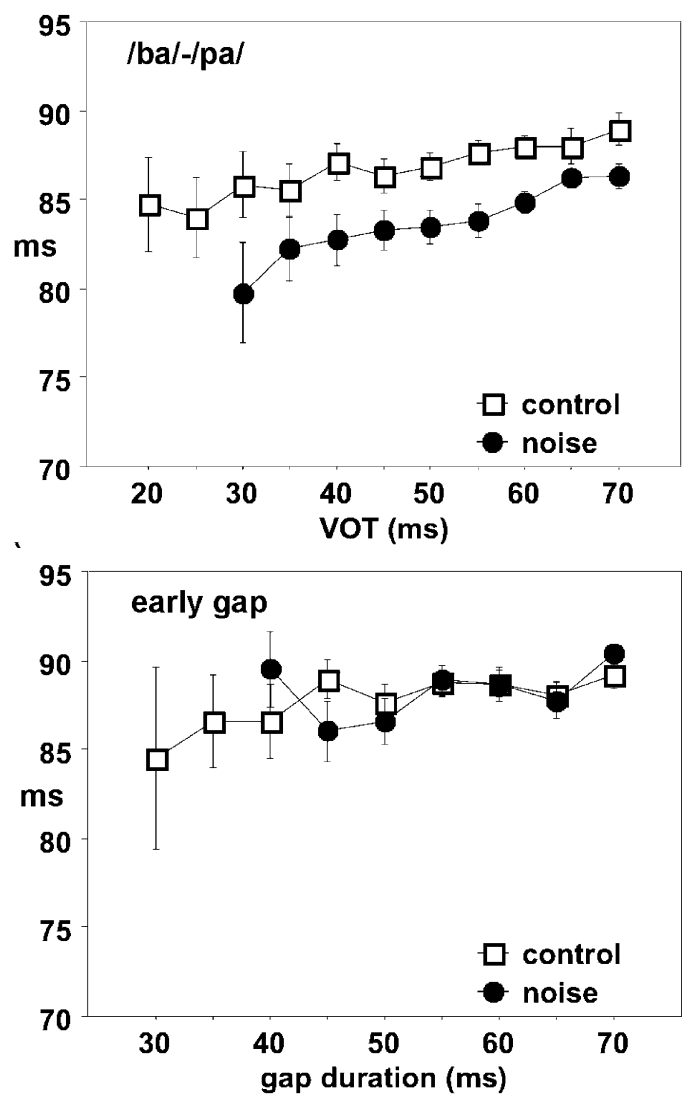

FIG. 5. Interpeak latency differences between the response to the leading noise burst and the vowel (top) and the trailing noise burst (bottom) for the control and noise-exposed groups. Error bars indicate standard deviation.

or trailing noise burst for each VOT or gap duration. Table 1 shows the minimum VOTs and early-gap durations for which the response to the vowel or trailing noise bursts was significantly larger $(p<0.01$, Bonferroni correction) than the spontaneous activity. There is a 10-ms increase for both minimum VOT and minimum early-gap duration in the noise-trauma animals. This finding was the same when calculated for averaged nonnormalized responses.

For $/ \mathrm{ba} /-/ \mathrm{pa} / \mathrm{stimuli}$, the width of the response peak to the leading noise burst was slightly larger for the noise-trauma animals compared to the controls, and the peak latency was also slightly increased. In contrast, the peak latency of the response to the vowel was slightly shortened, making the latency between the peak onset responses about 2-5 ms shorter in the trauma group compared to the control (Fig. 5). For the gap-in-noise stimuli, both the onset responses to leading and trailing bursts were shorter in the noise-trauma group, but no significant changes in the peak-to-peak response latency were observed.

Figure 6 shows the normalized $\mathrm{FR}_{\max }$ to the vowel or trailing noise burst as a function of VOT or gap duration. The normalized $\mathrm{FR}_{\max }$ as a function of VOT for the $/ \mathrm{ba} /-/ \mathrm{pa} /$ continuum in the noisetrauma group appeared steeper than that for controls but with the $50 \%$ point hardly changed. There were no significant differences in the normalized $\mathrm{FR}_{\text {max }}$ between the two curves. In contrast, the $\mathrm{FR}_{\text {max }}$ for the response to the trailing noise burst in the trauma group was significantly smaller ( $t$ test, $p<$ 0.01) than that in the control group for gap durations at 50, 60, 65, and $70 \mathrm{~ms}$; hence, the response curve was shallower compared to that for the control group.

\section{DISCUSSION}

We showed that mild hearing loss resulting from noise trauma induced in juvenile cats produced in adulthood a $10-\mathrm{ms}$ increase in the population minimum VOT. Concurrently, the $\mathrm{FR}_{\max }-\mathrm{VOT}$ function appeared steeper, but the $50 \%$ point remained unaffected. For the early gap-in-noise stimuli, we also found a $10-\mathrm{ms}$ increase in the minimum detectable gap, but here accompanied by a clear reduction in the slope of firing-rate-early-gap function. As a result,
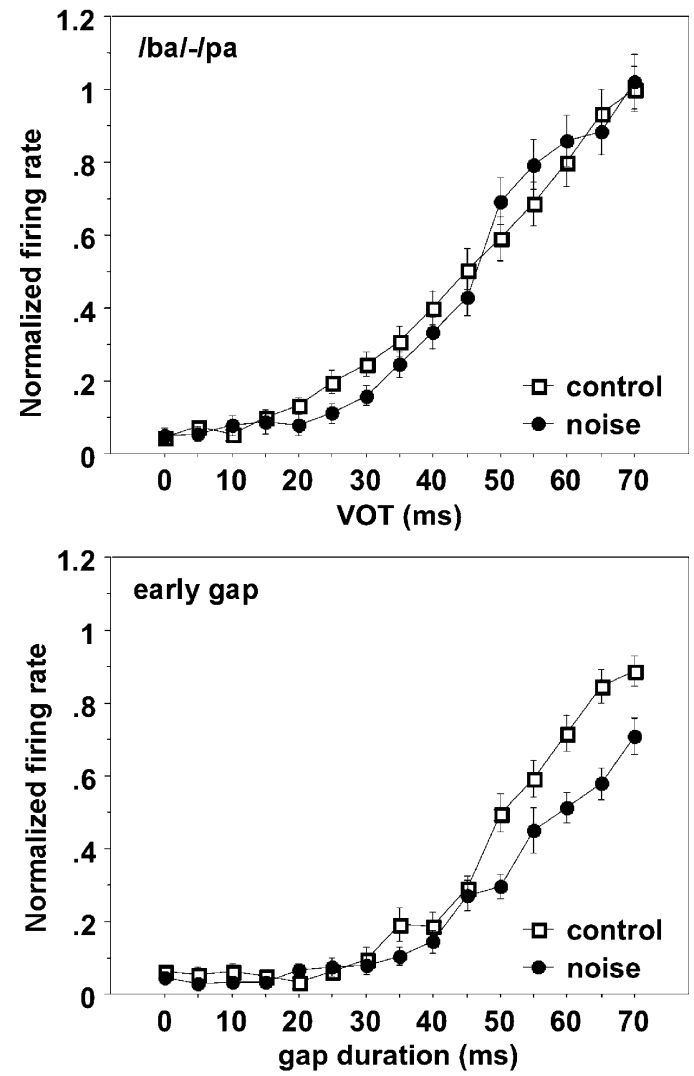

FIG. 6. Normalized peak firing rates for the response to the vowel (top) or trailing noise burst (bottom) for the controls and noiseexposed animals. 
the $50 \%$ point of the function also shifted by about $10 \mathrm{~ms}$. This shift could point to an increased postactivation suppression similar to that observed by Noreña et al. (2003) and Tomita et al. (2004) within a few hours after noise exposure.

Our finding that the $50 \%$ point of the response function describing the onset response to the vowel did not change after the noise-induced hearing loss may correspond to the absence of a phonetic boundary shift in children with mild to moderate hearing loss (Johnson et al. 1984). They also showed steeper labeling functions (\% correct) compared to the controls, as in our population data, but only in case of profound hearing impairment.

Our finding that the response function for the trailing noise burst in the "early-gap" stimuli showed a considerable shift of the $50 \%$ point and a decrease in the slope does suggest a potential decrease in the recovery from the leading noise burst-induced activity. Given that auditory perceptual decisions have to be based on activity in auditory cortex, and that the direct recordings from humans show that VOT is represented in primary auditory cortex (Steinschneider et al. 1999), one would expect some detrimental effect of perception. We have previously shown, and extensively modeled, that increasing the leading burst duration does not affect the recovery if expressed as a function of the onset interval between leading and trailing noise bursts in normal hearing cats (Eggermont 2000).

The apparent contradiction of increasing latency for the response to the noise burst and the decrease of the response latency to the vowel is resolved if one considers that the noise burst amplitude is about 10 $\mathrm{dB}$ lower than the peak vowel amplitude and will only be about $20 \mathrm{~dB}$ above the threshold. Therefore, the response latency to the noise burst of the $/ \mathrm{ba} /-/ \mathrm{pa} /$ stimuli increases. The reduced peak firing to the burst reduces the postactivation suppression, evident from the increase in spontaneous activity immediately following the onset response (Fig. 4). As a consequence, the onset response to the vowel occurs earlier compared to that in control cats. For the gap stimuli, the leading and trailing noise bursts have equal amplitude and also equal to that of the peak vowel amplitude and are thus, on average, $30 \mathrm{~dB}$ above threshold in the hearing-loss ears. Both onset response latencies were slightly reduced, likely as a result of increased feed-forward inhibition (Noreña et al. 2003), which eats away (because of the extra synaptic delay) on the longer latency parts of the onset response, thereby reducing its peak latency.

Six-week-old kittens do have mature hearing thresholds and cortical response latencies, but other cortical response properties, such as duration of the postactivation suppression and frequency-tuning curve bandwidth, remain immature up to 100-120 days of age (Eggermont 1996b), as are cortical frequency place maps (Bonham et al. 2004). Spontaneous firing rates take even longer to mature. Thus, at 6 weeks of age, the noise exposure was expected to affect the cochlea in about the same way as in an adult cat. It was surprising that the noise-induced hearing loss was frequency-independent over a wide range, albeit there was about $10 \mathrm{~dB}$ more hearing loss in the 16- to $24-\mathrm{kHz}$ range, as measured by $\mathrm{ABR}$, and up to $20 \mathrm{~dB}$ more above $16 \mathrm{kHz}$ as measured by multiunit thresholds in the cortex. This is different from that observed about $5 \mathrm{~h}$ after a noise trauma; here, the loss is negligible up to $4 \mathrm{kHz}$ and then rapidly increases to about $40 \mathrm{~dB}$ for frequencies above $6 \mathrm{kHz}$ (Noreña et al. 2003; Tomita et al. 2004). At longer times, after the trauma, the loss typically reduces somewhat but does not spread to frequencies below $4 \mathrm{kHz}$ (Seki and Eggermont 2002; Noreña and Eggermont 2005).

The effect of the ensuing hearing loss on the cortex may be different as the cortical frequency place maps are still changing at 6 weeks of age. In particular, the area representing units with CFs between 3 and $15 \mathrm{kHz}$ increases in size (Bonham et al. 2004). The cochlea is likely the only place for the origin of the extended frequency range of the hearing loss. We previously observed in adult cats the neurotoxic effect of noise exposure (Puel et al. 1998) for the frequency range above $12 \mathrm{kHz}$. This could be ameliorated by continuous exposure to moderately loud sound in that frequency range immediately following the noise trauma (Noreña and Eggermont 2005). If in young animals the frequency range where neurotoxic effects occur extends also to frequencies below the "trauma-tone" frequency, it would provide an explanation for the observed hearing loss in the low frequencies.

Another possibility would be that the noise produced only a minor high-frequency hearing loss, and that, in addition, a hearing loss of the conductive type was present. In that case, one expects that the latencies of the cortical responses would be longer in hearing-loss animals than in controls. Our data for gap stimuli show that the opposite occurred: the latencies to the leading burst were shorter in the noise-trauma group compared to the controls. Thus, it is unlikely that a conductive hearing loss was present. Therefore, the increase in thresholds (both from $\mathrm{ABR}$ and from cortical neurons) for frequencies as low as $1 \mathrm{kHz}$ must be particular to the age at which the animals were exposed. This has been noted before (Rybalko and Syka 2001).

Broadening of the frequency-tuning curve was restricted to the $\mathrm{CF}$ range between 10 and $20 \mathrm{kHz}$ and likely related to the increased threshold at $\mathrm{CF}$ in 
that region. This is comparable to the change found by Noreña et al. (2003), within a few hours after the noise trauma, in the $\mathrm{CF}$ range one octave above the trauma-tone frequency.

A model for the effect of the duration of the leading noise burst on the minimum detectable early gap (Eggermont 2000) predicts that increased postactivation suppression, as found within the first few hours after a noise trauma (Noreña et al. 2003), would shift the $\mathrm{FR}_{\max }$-gap duration curve to larger gap durations without affecting the shape thereof. Thus, one needs a postactivation suppression in the noise-exposed animals that becomes progressively stronger with time after the response to the leading noise burst to explain the observed changes. One such long latency and longer-lasting mechanism is related to the inhibitory action of $\mathrm{GABA}_{\mathrm{B}}$ receptors, resulting in long-lasting IPSPs. However, the effectiveness of $\mathrm{GABA}_{\mathrm{B}}$-ergic activity in the inferior colliculus decreased after noise-induced hearing loss (Szczepaniak and Møller 1995). As detailed in Buonomano and Merzenich (1998), one of the effects on $\mathrm{GABA}_{\mathrm{B}}$ receptors occurs in the form of a paired-pulse depression (PPD) of the fast IPSP in pyramidal cells; the other is in mediating the slow IPSP, which acts on a time scale following the fast IPSP. Thus, the two actions of the $\mathrm{GABA}_{\mathrm{B}}$-ergic system act in opposite ways. If we assume that after noise trauma, the effectiveness of $\mathrm{GABA}_{\mathrm{B}}$-ergic activity also decreases in auditory cortex, it would reduce the PPD of the fast IPSP and thus would make it stronger. This could explain the observed reduction in recovery at high gap durations, the less steep response-gap duration curve, and also the shift in MNGT.

Ketamine anesthesia has potentially a modest effect on the minimum gap values as discussed before (Eggermont 2000). We noted the comparison with the studies of Steinschneider et al. (1994), who recorded multiunit activity in awake monkey AI, and Steinschneider et al. (1999), who recorded local field potentials to VOT from human cortex. In these cases, the minimum VOT (for a double on-response) was very comparable. In double-click experiments in unanesthetized rabbit cortex, Fitzpatrick et al. (1999) found that a substantial fraction of neurons had recovery times as short as a few milliseconds, a large group of neurons had recovery times in the 20to 40-ms range similar to our results, and the remainder had longer recovery times. Thus, results could have been somewhat different in the awake animal, but the striking similarity of our findings with data recorded from awake human auditory cortex suggests that conclusions from our data can be extended to humans.

In summary, hearing loss induced by noise exposure in early age is of similar magnitude as in adult- exposed animals, but it covers a larger frequency range than in adult-exposed animals, particularly extending toward frequencies below the trauma frequency. The effect on minimum VOT and MNGT is also larger than in animals exposed to noise in adulthood. This suggests that hearing loss acquired in early age may affect cortical temporal processing in adulthood.

\section{ACKNOWLEDGMENTS}

This work was supported by the Alberta Heritage Foundation for Medical Research, the National Sciences and Engineering Research Council, the Canadian Language and Literacy Research Network, and the Campbell McLaurin Chair for Hearing Deficiencies. Greg Shaw provided programming support, and Boris Gourevitch helped with the figures.

\section{REFERENCES}

Bonham BH, Cheung SW, Godey B, Schreiner CE. Spatial organization of frequency response areas and rate/level functions in the developing AI. J. Neurophysiol. 91:841-854, 2004.

Buonomano DV, Merzenich MM. Net interaction between different forms of short-term synaptic plasticity and slow-IPSPs in the hippocampus and auditory cortex. J. Neurophysiol. 80:17651774, 1998.

EGGERMONT JJ. Representation of a voice onset time continuum in primary auditory cortex of the cat. J. Acoust. Soc. Am. 98:911920, 1995.

EgGERMONT JJ. How homogeneous is cat primary auditory cortex? Evidence from simultaneous single-unit recordings. Auditory Neurosci. 2:76-96, 1996a.

EgGermont JJ. Differential maturation rates for response parameters in cat primary auditory cortex. Auditory Neurosci. 2:309327, $1996 \mathrm{~b}$.

EGGERMONT JJ. Neural correlates of gap detection in three auditory cortical fields in the cat. J. Neurophysiol. 81:2570-2581, 1999.

EgGERMONT JJ. Neural responses in primary auditory cortex mimic psychophysical, across-frequency-channel, gap-detection thresholds. J. Neurophysiol. 84:1453-1463, 2000.

Fitzpatrick DC, Kuwada S, Kim DO, Parham K, Batra R. Responses of neurons to click-pairs as simulated echoes: auditory nerve to auditory cortex. J. Acoust. Soc. Am. 106:3460-3472, 1999.

Johnson D, Whaley P, Dorman MF. Processing of cues for stop consonant voicing by young hearing-impaired listeners. J. Speech Hear. Res. 27:112-118, 1984.

NGAN EM, MAY BJ. Relationship between the auditory brainstem response and auditory nerve thresholds in cats with hearing loss. Hear. Res. 156:44-52, 2001.

Noreña AJ, Eggermont JJ. Enriched acoustic environment after noise trauma reduces hearing loss and prevents cortical map reorganization. J. Neurosci. 25:699-705, 2005.

Noreña AJ, Tomita M, Eggermont JJ. Neural changes in cat auditory cortex after a transient pure-tone trauma. J. Neurophysiol. 90:2387-2401, 2003.

Puel JL, Ruel J, Gervais D’Aldin C, Pujol R. Excitotoxicity and repair of cochlear synapses after noise-trauma induced hearing loss. NeuroReport 9:2109-2114, 1998. 
Revoile S, Pickett JM, Holden-Pitt LD, Talkin D, Brandt FD. Burst and transition cues to voicing perception for spoken initial stops by impaired- and normal-hearing listeners. J. Speech Hear. Res. 30:3-12, 1987.

Ryвalko N, Syka J. Susceptibility to noise exposure during postnatal development in rats. Hear. Res. 155:32-40, 2001.

Seki S, Eggermont JJ. Changes in cat primary auditory cortex after minor-to-moderate pure-tone induced hearing loss. Hear. Res. 173:172-186, 2002.

Sharma A, Marsh CM, Dorman MF. Relationship between N1 evoked potential morphology and the perception of voicing. J. Acoust. Soc. Am. 108:3030-3035, 2000.

Steinschneider M, Schroeder C, Arezzo JC, Vaughan HG Jr. Speechevoked activity in primary cortex: effects of voice onset time. Electroencephalogr. Clin. Neurophysiol. 92:30-43, 1994.

Steinschneider M, Schroeder C, Arezzo JC, Vaughan HG Jr. Physiologic correlates of the voice onset time boundary in primary auditory cortex (A1) of the awake monkey: temporal response patterns. Brain Lang. 48:326-340, 1995.

Steinschneider M, Vokov IO, Noh MD, Garell PC, Howard MA III. Temporal encoding of the voice onset time phonetic parameter by field potentials recorded directly from human auditory cortex. J. Neurophysiol. 82:2346-2357, 1999.

Steinschneider M, Fishman YI, Arezzo JC. Representation of the voice onset time (VOT) speech parameter in population responses within primary auditory cortex of the awake monkey. J. Acoust. Soc. Am. 114:307-321, 2003.

Szczepaniak WS, Møller AR. Evidence of decreased GABAergic influence on temporal integration in the inferior colliculus following acute noise exposure: a study of evoked potentials in the rat. Neurosci. Lett. 196:77-80, 1995.

Tomita M, Noreña AJ, Eggermont JJ. Effects of an acute acoustic trauma on the representation of a voice onset time continuum in cat primary auditory cortex. Hear. Res. 193:39-50, 2004. 